\title{
Attitude Adaptive Control Of Combined Spacecraft
}

\author{
Qian Wang, Xinhong Li, Guanghong He, Zhibin Zhang, Jiping An \\ The Academy of Equipment, China, 3380 Huairou.\#95, Beijing, 101416 \\ 1258897329@qq.com
}

Keywords: combination of spacecraft; attitude; adaptive control.

\begin{abstract}
Aiming at the attitude adaptive control of spacecraft after docking, an adaptive attitude control method is proposed. Firstly, the dynamic modeling and spatial disturbance torque modeling of the combined spacecraft are carried out using the modified Rodriguez parameters to describe the attitude of the combined spacecraft. Secondly, according to the equation of attitude motion, the characteristic equation of closed-loop control system is deduced, and an adaptive attitude controller is designed. Finally, Matlab/Simulink simulation tool simulates the analysis. The simulation results show the effectiveness of the proposed controller for attitude control of the combined spacecraft.
\end{abstract}

\section{Introduction}

With the continuous innovation of the space technology and the improving ability of space research, development and application, the structure and composition of spacecrafts are becoming more and more complex. The performance and technology of the spacecrafts are continuously improved. In this case, the tradition single spacecraft has been unable to ensure it can be more lasting, stable and safe in orbit in the complex space environment. In order to solve this problem, researchers have proposed the implementation of assembly, repair and maintenance on orbit and other on orbit servicing technologies ${ }^{[1]}$. In the process of orbital service, the safe and stable operation of the combined spacecraft which make up of the service spacecraft and the target spacecraft is the prerequisite and key to the success of the on-orbit service.

Aiming at the attitude control of spacecraft, a lot of researches have been done by domestic and foreign scholars. But the study for the combination of spacecraft attitude adaptive control is relatively weak, in this showtime, Xie Yongchun et al design a attitude controller for different configurations (straight and L-shaped) in combination spacecraft based on the intelligent algorithm of the features model $^{[2]}$, Northwestern Polytechnical University, Wang et al proposed a coordination based on the combined spacecraft attitude control method of the robot arm coupling torque assessment $^{[3]}$, this approach can achieve the attitude stabilization of combined spacecraft only through space manipulator and reaction flywheel, but the method is complication and the computation is large, it also did not consider disturbance torque in the control process; Schaub and Junkins use a modified Rodrigues parameters proposed a global asymptotically stable attitude control scheme $e^{[4]}$, it is also not taken into account the effect of interference torque. Reference [5] studied the optimal control of spacecraft, but did not consider the impact of interference torque. Reference [6] uses a fuzzy control method to study attitude adjustment problems of spacecraft existing the interference torque and inertia uncertain. Reference [7] considers spacecraft attitude control with disturbance torque, but this paper uses the singular values of Euler angles to describe spacecraft attitude motion.

For the spacecraft in orbit, it will inevitably be affected by a variety of torque interference, these moments mainly include gravity gradient torque, solar pressure moment and aerodynamic torque. These disturbing moments have a great impact on the design of spacecraft attitude control scheme.

Therefore, in this paper, we will model the combined spacecraft based on the attitude kinematics and attitude dynamics model of the spacecraft, which is described by the modified Rodriguez parameter without singular value, and propose an attitude adaptive control method for the combined spacecraft, and verify its feasibility through Matlab/Simulink simulation tools.

\section{Combined Spacecraft Modeling}

\subsection{Dynamic Modeling}

Attitude dynamic equation is an equation describes the variation of attitude parameters with time in the course of spacecraft maneuvering. There are varities of forms to describe the parameters of spacecraft attitude, considering the advantages and disadvantages of the various parameters, we describe spacecraft attitude kinematics by the modified Rodriguez parameter ${ }^{[8]}$.

Rodriguez parameters is a minimal description of the attitude parameter method. It is defined as:

$$
r=\left[\begin{array}{lll}
r_{1} & r_{2} & r_{3}
\end{array}\right]^{\mathrm{T}}=e \tan (\theta / 2)
$$

Where the scalars $e$ and $\theta$ are the Euler rotation axis and Euler rotation angle ${ }^{[9][10]}$.

But from the definition of the Rodriguez parameters we can find singular when the rotation angle is 180 . The modified Rodriguez parameter (MRP) can avoid this problem, which is defined as 


$$
r=\left[\begin{array}{lll}
r_{1} & r_{2} & r_{3}
\end{array}\right]^{\mathrm{T}}=e \tan (\theta / 4)
$$

As in equation (1), $e$ is the Euler axis, $\theta$ is the Euler rotation angle.

Here we define a vector $x=\left[\begin{array}{lll}x_{1} & x_{2} & x_{3}\end{array}\right]^{\mathrm{T}} \in \mathrm{R}^{3}$, and the cross-product matrix corresponding to the vector is denoted by $\tilde{x}$

$$
\tilde{x}=\left[\begin{array}{ccc}
0 & -x_{3} & x_{2} \\
x_{3} & 0 & -x_{1} \\
-x_{2} & x_{1} & 0
\end{array}\right\rfloor
$$

Use of MRP to describe spacecraft attitude dynamic equation:

$$
\dot{r}=G(r) \omega
$$

among them, $r=\left[\begin{array}{lll}r_{1} & r_{2} & r_{3}\end{array}\right]^{\mathrm{T}} \in \mathrm{R}^{3}$ is MRP of spacecraft; $\omega=\left[\begin{array}{lll}\omega_{1} & \omega_{2} & \omega_{3}\end{array}\right]^{\mathrm{T}}$ is angular velocity of the attitude of the spacecraft;

$$
G(r)=\frac{1}{4}\left[\left(1-r^{\mathrm{T}} r\right) E_{3 \times 3}+2 \tilde{r}+2 r r^{\mathrm{T}}\right]^{\mathrm{T}}
$$

In the above equation, $E_{3 \times 3}$ is unit matrix, $\tilde{r}$ is cross-product matrix.

The attitude dynamics equation of rigid spacecraft is ${ }^{[11]}$ :

$$
I \dot{\omega}=-\tilde{\omega} I \omega+T_{u}+T_{d}
$$

among them, $\omega=\left[\begin{array}{lll}\omega_{1} & \omega_{2} & \omega_{3}\end{array}\right]$ is the attitude of the spacecraft angular velocity, $I$ is the spacecraft inertia, $\tilde{\omega}$ is a cross-product matrix. $T_{u}$ is control torque and $T_{d}$ is the disturbance torque.

It is assumed that the spacecraft body coordinate system is $O_{s}-X_{s} Y_{s} Z_{s}$, the target spacecraft is $O_{t}-X_{t} Y_{t} Z_{t}$, and the coordinate axis coincides with the inertial principal axis. The known service spacecraft is of mass $m_{s}$, the rotational inertia is $I_{s}=\operatorname{diag}\left\lfloor I_{s x}, I_{s y}, I_{s z}\right\rfloor$; The mass of the target spacecraft is $m_{t}$, The rotational inertia is $I_{t}=\operatorname{diag}\left\lfloor I_{t x}, I_{t y}, I_{t z}\right\rfloor$, as shown in Figure 1,

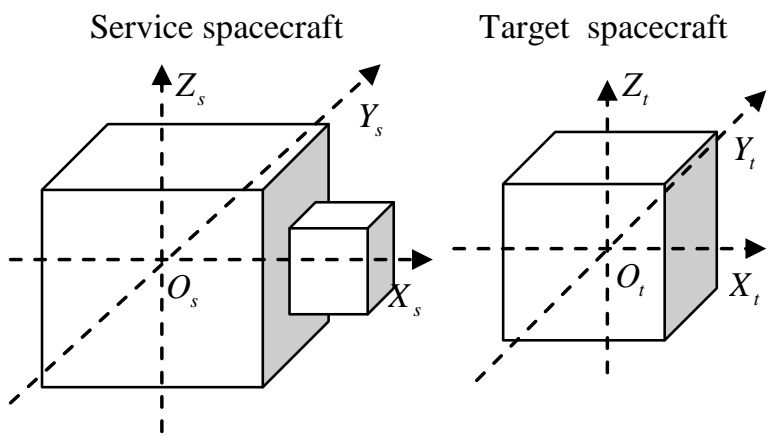

Figure 1:Body coordinate system of spacecraft before docking After the docking is completed, set $O_{s}-X_{s} Y_{s} Z_{s}$ the coordinate system as the reference coordinate system, versus the relative position vector between $O_{t}$ and $O_{s}$ is $r_{t s}=\left[\begin{array}{lll}x & y & z\end{array}\right]^{\mathrm{T}}$. the coordinate transformation matrix of $O_{t}-X_{t} Y_{t} Z_{t}$ to $O_{s}-X_{s} Y_{s} Z_{s}$ is $R_{t}^{s}$,accordance with the order of 1-2-3 rotation $\varphi_{0}, \theta_{0}, \psi_{0}$

$$
\begin{gathered}
R_{t}^{s}=\left[\begin{array}{ll}
\cos \psi_{0} \cos \theta_{0} & \sin \psi_{0} \cos \varphi_{0}+\cos \psi_{0} \sin \theta_{0} \sin \varphi_{0} \\
-\sin \psi_{0} \cos \theta_{0} & \cos \psi_{0} \cos \varphi_{0}-\sin \psi_{0} \sin \theta_{0} \sin \varphi_{0} \\
\sin \theta_{0} & -\cos \theta_{0} \sin \varphi_{0}
\end{array}\right] \\
\sin \psi_{0} \sin \varphi_{0}-\cos \psi_{0} \sin \theta_{0} \cos \varphi_{0} \\
\cos \psi_{0} \sin \varphi_{0}+\sin \psi_{0} \sin \theta_{0} \cos \varphi_{0} \\
\cos \theta_{0} \cos \varphi_{0}
\end{gathered}
$$

Available make

$$
R_{t}^{s}=\left\lfloor\begin{array}{lll}
a_{11} & a_{12} & a_{13} \\
a_{21} & a_{22} & a_{23} \\
a_{31} & a_{32} & a_{33}
\end{array}\right\rfloor
$$

Convert $I_{t}$ to the reference coordinate system

$$
I_{t}{ }^{\prime}=R_{t}^{s} I_{t}\left(R_{t}^{s}\right)^{\mathrm{T}}
$$

The inertia of the combined spacecraft $I$ in the reference coordinate system is

$$
\begin{aligned}
& I=I_{s}+I_{t}^{\prime}+m_{t}\left(r_{t s}^{\mathrm{T}} r_{t s} E-r_{t s} r_{t s}^{\mathrm{T}}\right)- \\
& \frac{\left(m_{t} r_{t s}\right)^{\mathrm{T}}\left(m_{t} r_{t s}\right) E-\left(m_{t} r_{t s}\right)\left(m_{t} r_{t s}\right)^{\mathrm{T}}}{m_{s}+m_{t}}
\end{aligned}
$$

In general, it is assumed that $O_{s} X_{s}$ coincides with $O_{t} X_{t}$ for convenience of control convenience, $r_{t s}=\left[\begin{array}{lll}x & 0 & 0\end{array}\right]^{\mathrm{T}}$, $\theta_{0}=\psi_{0}=0$; and $R_{t}^{s}$ Can be simplified as

$$
R_{t}^{s}=\left\lfloor\begin{array}{ccc}
1 & 0 & 0 \\
0 & a_{22} & a_{23} \\
0 & a_{32} & a_{33}
\end{array}\right\rfloor=\left[\begin{array}{ccc}
1 & 0 & 0 \\
0 & \cos \varphi_{0} & \sin \varphi_{0} \\
0 & -\sin \varphi_{0} & \cos \varphi_{0}
\end{array}\right\rfloor
$$

Service spacecraft Target spacecraft

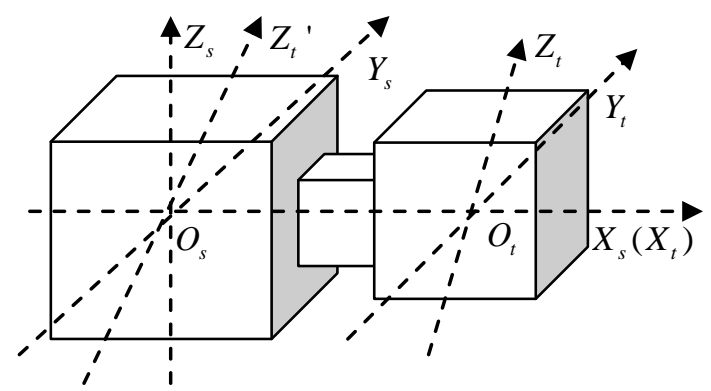

Figure 2: Body coordinate system of spacecraft after docking Substituting equation 6,8 and simplified $r_{t s}$ into equation 9:

$$
\begin{aligned}
& I= \begin{cases}I_{s x}+I_{t x} & 0 \\
0 & I_{s y}+a_{22}^{2} I_{t y}+a_{23}^{2} I_{t z}+m^{\prime} x^{2} \\
0 & a_{22} a_{23} I_{t y}+a_{23} a_{33} I_{t z}\end{cases} \\
& \begin{array}{l}
0 \\
a_{22} a_{23} I_{t y}+a_{23} a_{33} I_{t z}
\end{array} \\
& I_{s z}+a_{32}^{2} I_{t y}+a_{33}^{2} I_{t z}+m^{\prime} x^{2}
\end{aligned}
$$


In the equation, $m^{\prime}=m_{t}\left(1-\frac{m_{t}}{m_{s}+m_{t}}\right)$.

off-diagonal elements in equation (10) right matrix

$$
\begin{aligned}
a_{22} a_{23} I_{t y}+a_{23} a_{33} I_{t z} & =-\cos \varphi_{0} \sin \varphi_{0} I_{t y}+\sin \varphi_{0} \cos \varphi_{0} I_{t z} \\
& =\sin \varphi_{0} \cos \varphi_{0}\left(I_{t z}-I_{t y}\right)
\end{aligned}
$$

In general, the spacecraft is designed to be an axisymmetric body and the axis of symmetry is $O_{t} X_{t}$ axis, means $I_{t y}=I_{t z}$. The non-diagonal elements in the equation are equal to zero, then the inertia matrix of the combined spacecraft can be obtained by:

$$
\begin{aligned}
& I=\left\lfloor\begin{array}{ll}
I_{s x}+I_{t x} & 0 \\
0 & I_{s y}+a_{22}^{2} I_{t y}+a_{23}^{2} I_{t z}+m^{\prime} x^{2} \\
0 & 0
\end{array}\right. \\
& 0 \\
& 0 \\
& I_{s z}+a_{32}^{2} I_{t y}+a_{33}^{2} I_{t z}+m^{\prime} x^{2}
\end{aligned}
$$

\subsection{Disturbing Torque Modeling}

Spacecraft in orbit is inevitably subject to a variety of interference torque, these torques include aerodynamic torque, gravitational gradient torque, solar interference torque and magnetic interference torque. In this paper, the aerodynamic torque, the gravitational gradient torque and solar interference torque will be modeled and analysed.

\subsubsection{Aerodynamic Torque}

Aerodynamic interference force and aerodynamic disturbance torque are given by following formula ${ }^{[12]}$ :

$$
F_{p}=-\frac{1}{2} c_{d} \cdot \rho \cdot v^{2} \cdot A
$$

$c_{d}$ is the aerodynamic drag coefficient, generally between 2.2 and 2.4, herein take 2.2; $v$ is the velocity of spacecraft relative to atmosphere; $A$ is the equivalent area of spacecraft affected by atmosphere; $\rho$ is the atmospheric density of spacecraft's altitude.

The calculation of aerodynamic moment must first calculate the atmospheric density, which is related to the orbit height of spacecraft. Generally, the atmospheric density is calculated by the exponential model ${ }^{[13]}$ :

$$
\rho=\rho_{0} \exp \left(-\frac{h-h_{0}}{H}\right)
$$

Wherein $\rho_{0}$ and $h_{0}$ is the reference density and the reference height, $h$ is the spacecraft orbital altitude, $H$ is the segment scale height.

$$
T_{d p}=L_{a} \times F_{p}
$$

$L_{a}$ is the radius vector of the center of windward side's pressure relative to the aircraft's barycentre.

\subsubsection{Gravity Gradient Torque}

Because the spacecraft is in the gravitational field of the center of earth, each tiny mass element is affected by the gravity, the gravitational distribution is related to the on-orbit attitude of the spacecraft. The resultant force of gravity does not always pass through the center of mass of the spacecraft, And the resulting torque is the gravitational gradient torque. The gravitational gradient torque is calculated as follows ${ }^{[14]}$ :

$$
T_{d g}=\frac{3 \mu}{\|r\|^{5}} \tilde{r} I r
$$

In the formula, $\mu$ is the gravitational constant, $\mu=3.986 \times 10^{5} \mathrm{~km}^{3} / \mathrm{s}^{2} ; \quad r$ is the position vector of the spacecraft; $I$ is the rotational inertia of the spacecraft; $\tilde{r}$ is a cross-product matrix.

\subsubsection{Solar Pressure Torque}

The calculation of the solar radiation torque needs take the shadowing problem into account. As the sun is relatively far from the earth, for low-orbit spacecraft, the sunlight can be processed into parallel light and the shadow can be processed into a column. As shown in Figure3.

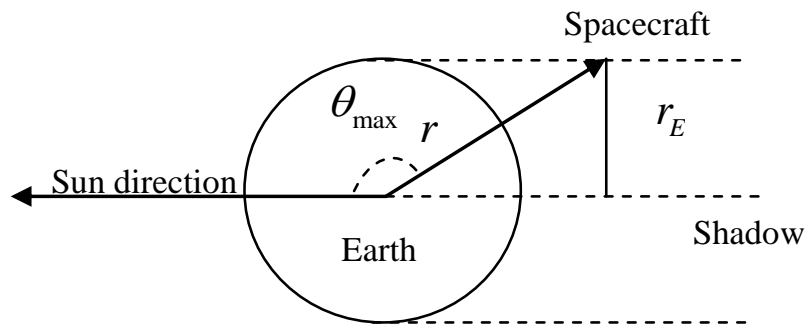

Figure 3: Spacecraft in the shadow boundary

It is obvious that if the spacecraft is exposed to the sun, the angle between the geocentric to solar vector and the geocentric to spacecraft vector must be less than $\theta_{\max }$, otherwise the spacecraft is not exposed to the sun, of which:

$$
\theta_{\max }=\frac{\pi}{2}+\arccos \left(\frac{r_{E}}{r}\right)
$$

In the formula, $r_{E}$ is the radius of earth, $r$ is the geocentric distance of spacecraft.

The solar radiation intensity near earth is $p$, the affect of solar radiation pressure is related to the surface material on the spacecraft, after the photon stream collide with the spacecraft surface, there will be absorption, specular reflection and scattering three forms and mixed forms of these forms ${ }^{[15]}$. In order to facilitate the analysis of the problem, we assume the photon stream is totally absorbed.
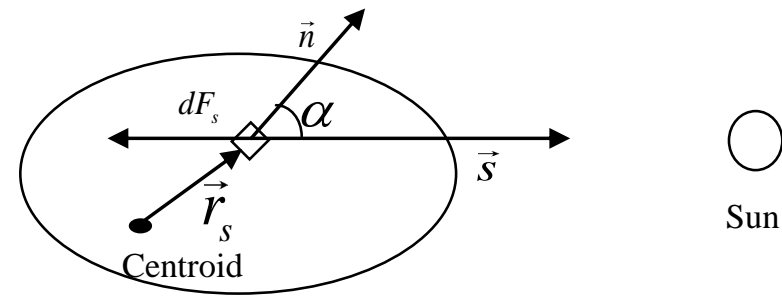

Sun

Figure 4: Spacecraft on the surface of infinitesimal pressure 
As shown in Figure4, $d S$ is the infinitesimal of the spacecraft surface, $\vec{n}$ is a normal vector which is perpendicular to the infinitesimal of the spacecraft surface, $\vec{r}_{s}$ is the position vector of the infinitesimal, $\vec{s}$ is the direction vector of sun, the projection area of the surface element is denoted as $d A$, then

$$
d A=\cos \alpha d S=\vec{n} \cdot \vec{s} d S
$$

The sunlight pressure of the infinitesimal is:

$$
d F=-p d A \vec{s}=-(p \vec{n} \cdot \vec{s} d S) \vec{s}
$$

The torque relative to spacecraft centroid is:

$$
d T=r_{s} \times d F=-p r_{s} \times \vec{s}(\vec{n} \cdot \vec{s}) d S
$$

Then the overall torque affected by the sun light pressure is:

$$
T_{d s}=\int_{S_{w s}} d T=-p \int_{S_{w s}} r_{s} \times \vec{s}(\vec{n} \cdot \vec{s}) d S
$$

Wherein $S_{w s}$ is the sunny side area of spacecraft.

\section{Adaptive Attitude Controller Design}

This section is based on the above two models, put forward an adaptive attitude control method, and designs an adaptive attitude controller. A centralized control strategy ${ }^{[16]}$ by the service spacecraft for combined spacecraft attitude control is presented, from the attitude kinematics equation of spacecraft(4) we can obtain:

$$
\ddot{r}=\dot{G}(r) \omega+G(r) \dot{\omega}
$$

In the above equation

$$
\dot{G}(r)=\frac{1}{4}\left[-\left(\dot{r}^{\mathrm{T}} r+r^{\mathrm{T}} \dot{r}\right) E_{3 \times 3}+2 \tilde{\dot{r}}+2 \dot{r} r^{\mathrm{T}}+2 r \dot{r}^{\mathrm{T}}\right]
$$

Design the following control law:

$$
\ddot{r}=-k r-p \dot{r}
$$

Then the characteristic equation of closed-loop control system is obtained:

$$
\ddot{r}=-k r-p \dot{r}
$$

The (4) and (20) into equation (23) is obtained:

$$
G(r)\left[\dot{\omega}+p \omega+G^{-1}(r)[\dot{G}(r) \omega+k r]\right]=0
$$

In the above formula for

$$
G^{-1}(r)=\left[1 /\left(1+r^{\mathrm{T}} r\right)^{2}\right] G^{\mathrm{T}}(r)
$$

and $G(r)$ is positive definite matrix, the equation (24)can be expressed as:

$$
\dot{\omega}+p \omega+G^{-1}(r)[\dot{G}(r) \omega+k r]=0
$$

which is

$$
\dot{\omega}=-p \omega-G^{-1}(r)[\dot{G}(r) \omega+k r]
$$

The virtual control $u$ is introduced here:

$$
u=-p \omega-G^{-1}(r)[\dot{G}(r) \omega+k r]
$$

When there is a gesture tracking instruction $r_{0}$, then

$$
u=-p \omega-G^{-1}(r)\left[\dot{G}(r) \omega+k\left(r-r_{0}\right)\right]
$$

The control torque is

$$
T_{u}=I u
$$

\section{Simulation Analysis}

The simulation model is built based on Matlab/Simulink simulation environment, as shown in Figure5, to provide the service spacecraft attitude control simulation model with the top-level interface.

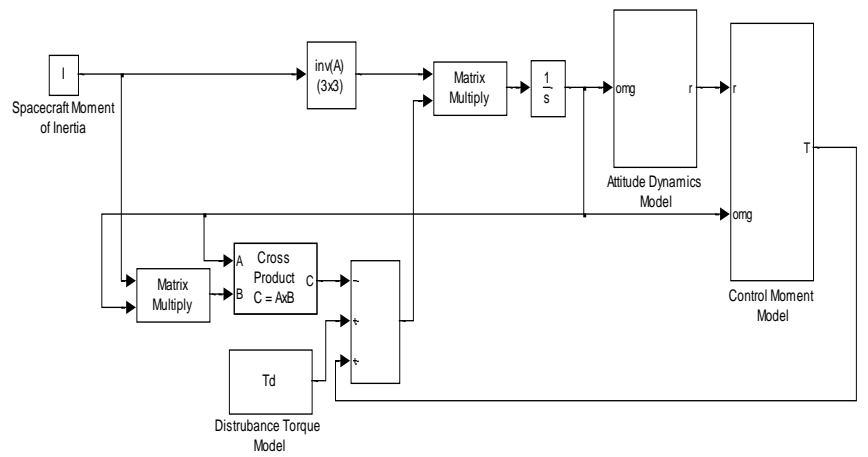

Figure 5: Spacecraft attitude control Simulink model

Assume that the orbit of the spacecraft is a circular orbit of a height of $500 \mathrm{~km}$. The inertia of the service spacecraft is $I_{s}=\operatorname{diag}[50,70,80] \mathrm{kg} \cdot \mathrm{m}^{2}$, the mass is $m_{s}=150 \mathrm{~kg}$. The inertia of the target spacecraft is $I_{t}=\operatorname{diag}[50,30,30] \mathrm{kg} \cdot \mathrm{m}^{2}$, the mass is $m_{t}=150 \mathrm{~kg}$. The effective area of the combined spacecraft affected by the atmosphere is $3 \mathrm{~m}^{2}$, the spacecraft sunny area is $10 \mathrm{~m}^{2}$; The relative position vector between the two spacecraft is $r_{t s}=\left[\begin{array}{ccc}2 & 0 & 0\end{array}\right]^{\mathrm{T}}, \theta_{0}=\varphi_{0}=0, \psi_{0}=5^{\circ}$; For the initial MRP is $r=\left[\begin{array}{lll}-0.03 & 0.4 & 0.2\end{array}\right]^{\mathrm{T}}$; Initial angular velocity is $\omega=\left[\begin{array}{lll}0.1 & 0.5 & 0.3\end{array}\right]^{\mathrm{T}} \mathrm{rad} / \mathrm{s}$; Combined spacecraft attitude parameters desired for the MRP is $r=\left[\begin{array}{lll}0.5 & 0.2 & 0.3\end{array}\right]^{\mathrm{T}}$. Controlled parameters $k=\operatorname{diag}[2,2,2]$, $p=\operatorname{diag}[6,6,6] ;$ The maximum control torque is $10 \mathrm{~N} \cdot \mathrm{m}$.

The combination of spacecraft attitude MRP, angular velocity and control torque curve of the combined spacecraft are shown in Figure6,7 and 8:

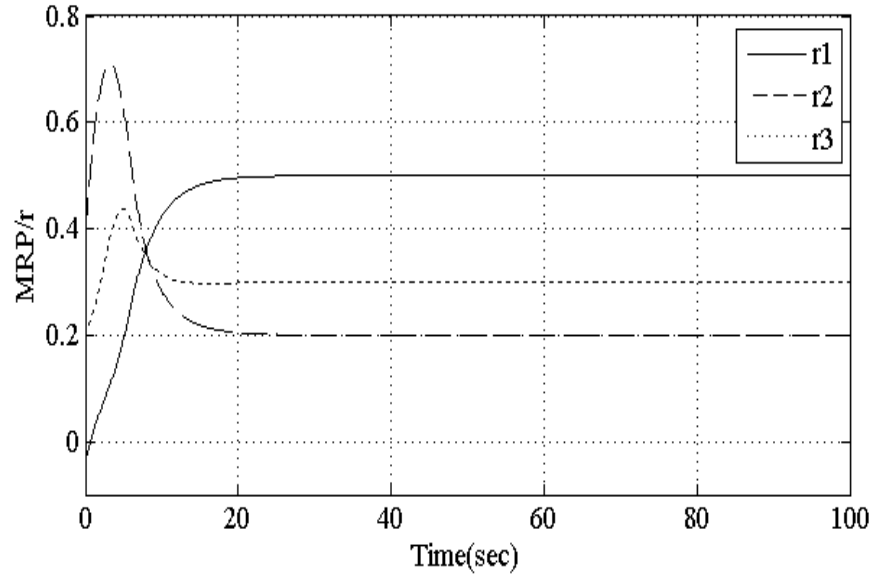

Figure 6: Combined Spacecraft Attitude MRP Variations 


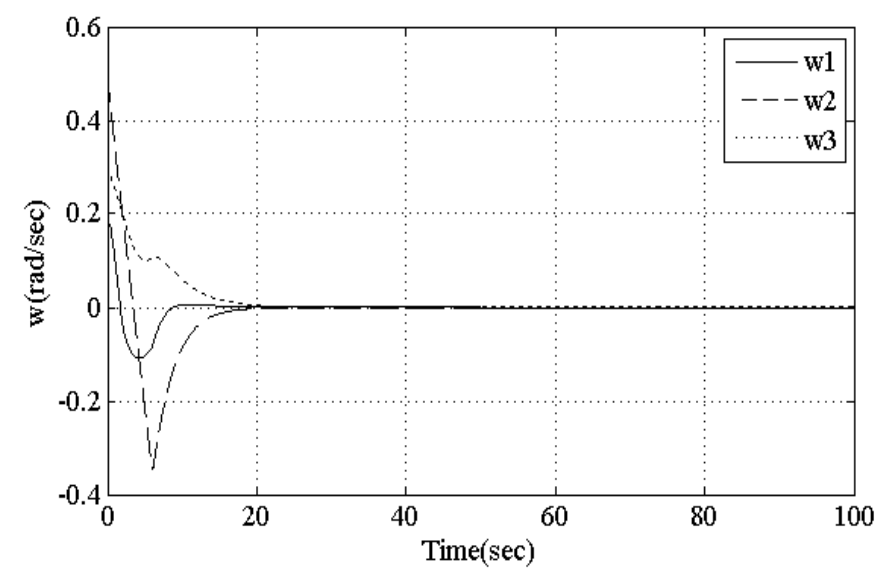

Figure 7: Combined Spacecraft Attitude Angular Velocity

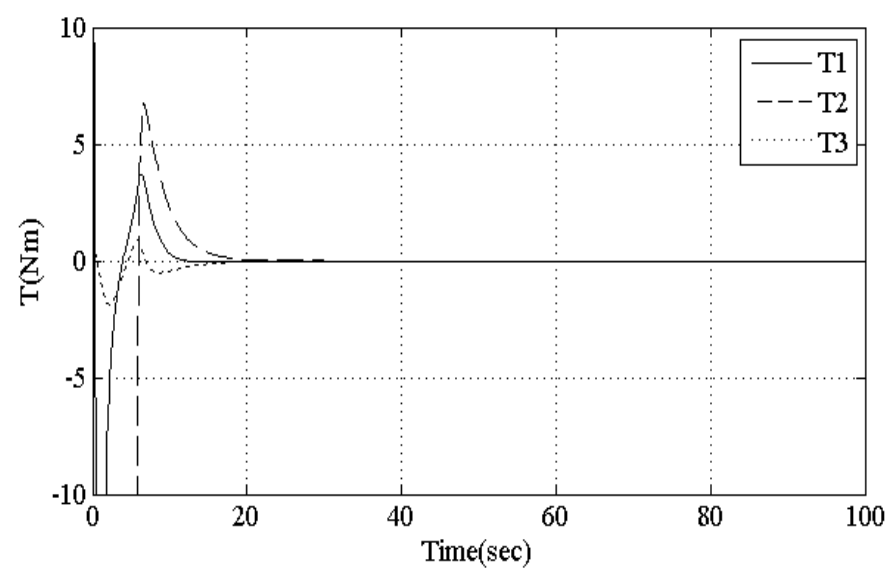

Figure 8: Combination Spacecraft Control Torque

Simulation results above show that the control law designed in equation (23) can adjust the attitude of the spacecraft to the desired attitude more quickly, and can be applied to the attitude control of spacecraft docking combined spacecraft, which has strong promotional.

\section{Conclusion}

In this paper, an adaptive attitude controller is designed to ensure the attitude stability of two spacecrafts after docking, and the attitude of the combined spacecraft is effectively controlled. The aerodynamic moment, the gravitational gradient moment and the solar light pressure moment are modeled and analyzed considering the external disturbance of the spacecraft in the space environment; and the rotation of the combined spacecraft is established due to the change of the inertia after the spacecraft combination Inertia model. Finally, the simulation results show that the controller can realize the attitude control of the combined spacecraft. The controller designed in this paper has simple structure and fast response, which can provide a reference for the attitude control of the combined spacecraft.

In this paper, we study the attitude vacancy of the combined spacecraft under the condition that the quality parameters of the service spacecraft and the target spacecraft are known. So we can further study the attitude control under the uncertain quality parameters of the combined spacecraft .

\section{References}

[1] Cui NaiGang, Wang Ping, Guo Jifeng, Cheng Xing. A review of on-orbit servicing [J]. Journal of Astronautics, 28, pp. 805-811, (2007).

[2] Yu Xinxin, Xie Yongchun. The attitude control of assembled spacecraft by using intelligent adaptive control method $[\mathrm{J}]$. Aerospace Control and Application, 35, pp. 36-41, (2009).

[3] Wang Ming, Huang Panfeng, Chang Haitao, Wang Dongke. Coordinated attitude control of combined spacecraft based on estimated coupling torque of manipulatior [J]. Robot, 37, pp. 25-34, (2015).

[4] H. Schaub and JL Junkins, "Stereographic orientation Parameters for Attitude Dynamics: A Generalization of the Rodrigues Parameters," Journal of the Astronautical Sciences, 44, pp. 1-19, (1996).

[5] Ei-Cohary A. Optimal control of a rigid spacecraft programmed motion without angular velocity measurements $[\mathrm{J}]$. European Journal of Mechanics A Solids, 25, pp.854-866, (2006).

[6] Song B, Ma G, Li C. Robust fuzzy controller design for a rigid spacecraft attitude regulation system [C] Proceeding of 1st International Symposium on Systems and Control in Astronautics Harbin, pp. 424-429, (2006).

[7] CHEN Jin-li, LI Dong-hai, SUN Xian-fang. Nonlinear robust decentralized controller design for spacecraft control system [J]. Journal of Astronautics, 27, pp. 6-11, (2006).

[8] Crassidis JL, Markley F. L. Sliding mode control using modified Rodrigues parameters [J]. Journal of Guidance, Control, and Dynamics, 19, pp. 1381-1383, (1996).

[9] H. Schaub and JL Junkins, Analytical Mechanics of Space System. Reston, VA: AIAA Education Series, (2009).

[10] H. Schaub and JL Junkins, "Stereographic orientation Parameters for Attitude Dynamics: A Generalization of the Rodrigues Parameters," Journal of the Astronautical Sciences, 32, pp. 578-586, (1996).

[11] James R. Wertz. Fundamentals of Spacecraft Attitude Determination and Control [M] Springer New York Heidelberg Dordrecht London, pp. 290, (2014).

[12] Hu Qizheng, Yang Fang. An introduction to astronautics [M]. Beijing: Science and Technology of China press, pp. 43 , (2010).

[13] JI Rongfen. Earth perturbation atmosphere model [J] Spacecraft Recovery and Remote Sensing, 6, pp. 11-13, (1995).

[14] Zhang Renwei. Satellite orbit attitude dynamics and control $[\mathrm{M}]$. Beijing: Beihang University Press: pp. 182, (2006).

[15] Liu Tun, Zhao Zhiping. Method to calculate effective area used in computation of solar radiation torque of satellite [J]. Journal of Nanjing University of Science and Technology, 31, pp. 84-88, (2007).

[16] TU XuYan, Wang Cong, Guo Yanhui. Large systems cybernetics [M]. Beijing: Beijing University of Posts and Telecommunications Press,( 2005). 